\title{
Atomic parity violation in a single trapped radium ion
}

\author{
O. O. Versolato · L. W. Wansbeek • G. S. Giri • J. E. van den Berg • \\ D. J. van der Hoek • K. Jungmann • W. L. Kruithof • C. J. G. Onderwater • \\ B. K. Sahoo • B. Santra • P. D. Shidling • R. G. E. Timmermans • \\ L. Willmann • H. W. Wilschut
}

Published online: 15 April 2011

(C) The Author(s) 2011. This article is published with open access at Springerlink.com

\begin{abstract}
Atomic parity violation (APV) experiments are sensitive probes of the electroweak interaction at low energy. These experiments are competitive with and complementary to high-energy collider experiments. The APV signal is strongly enhanced in heavy atoms and it is measurable by exciting suppressed (M1, E2) transitions. The status of APV experiments and theory are reviewed as well as the prospects of an APV experiment using one single trapped $\mathrm{Ra}^{+}$ion. The predicted enhancement factor of the APV effect in $\mathrm{Ra}^{+}$is about 50 times larger than in Cs atoms. However, certain spectroscopic information on $\mathrm{Ra}^{+}$needed to constrain the required atomic many-body theory, was lacking. Using the AGOR cyclotron and the TRI $\mu \mathrm{P}$ facility at KVI in Groningen, short-lived ${ }^{212-214} \mathrm{Ra}^{+}$ions were produced and trapped. First ever excited-state laser spectroscopy was performed on the trapped ions. These measurements provide a benchmark for the atomic theory required to extract the electroweak mixing angle to sub-1\% accuracy and are an important step towards an APV experiment in a single trapped $\mathrm{Ra}^{+}$ion.
\end{abstract}

Keyword Atomic parity violation

\section{Introduction}

APV experiments [1-10] are sensitive probes of the electroweak interaction at low energy. APV is due to the exchange of the $Z^{0}$ boson between the electrons and

FOM projectruimte 06PR2499, NWO Toptalent, ITS LEIF.

O. O. Versolato $(\bowtie) \cdot$ L. W. Wansbeek · G. S. Giri · J. E. van den Berg .

D. J. van der Hoek · K. Jungmann · W. L. Kruithof · C. J. G. Onderwater .

B. K. Sahoo · B. Santra · P. D. Shidling · R. G. E. Timmermans ·

L. Willmann · H. W. Wilschut

Kernfysisch Versneller Instituut, University of Groningen,

Zernikelaan 25, 9747 AA Groningen, The Netherlands

e-mail: versolato@kvi.nl 


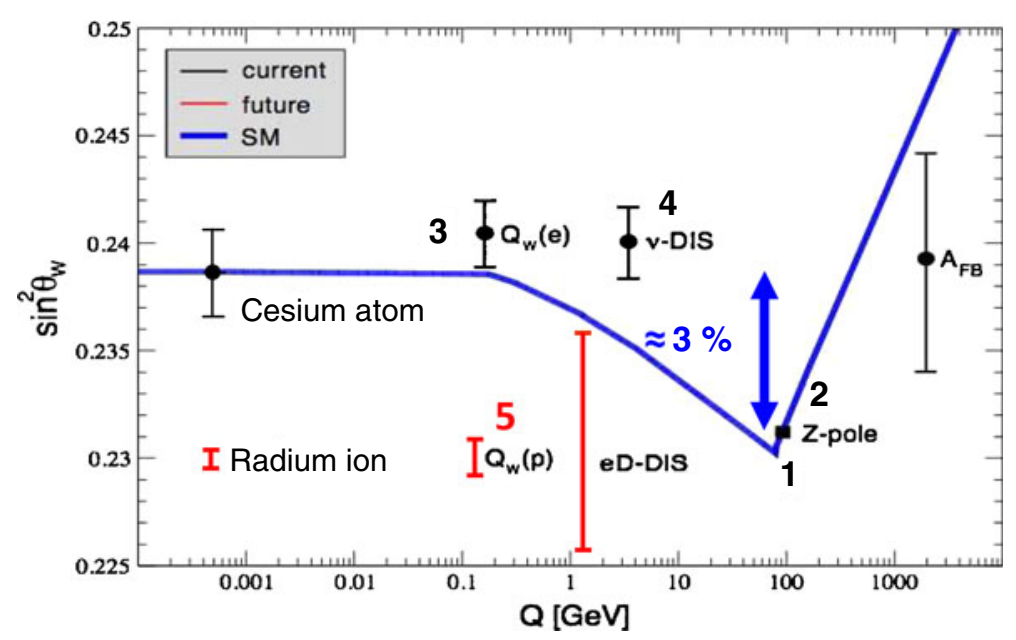

Fig. 1 Running of the electroweak coupling constant. Figure adapted from [15]. The blue line depicts the SM prediction of the running of the Weinberg angle. The black dots are actual measured points. The red lines indicate planned experiments with predicted error bars

the quarks in the atomic nucleus, which has effective weak charge $\left(\mathrm{Q}_{\text {weak }}\right)$. Its size depends on the mixing (or: Weinberg) angle of the photon and the $Z^{0}$ boson, which is a fundamental parameter of the electroweak theory. The Standard Model of particle physics (SM) makes a detailed but poorly tested prediction of the variation of the Weinberg angle with the energy with which it is probed. Experiments have been performed at high energy (near the $Z^{0}$-pole), medium, and low energy (cf. Fig. 1). The excellent agreement with the SM at low energy was only quite recently found after decades of tremendous theoretical efforts interpreting the original experiment [11]. Therefore, it is highly desirable to perform a competitive experiment at low energy. APV experiments have been of key importance in the acceptance of the electroweak theory, confirming together with neutrino scatting experiments [12] the existence of neutral currents over many orders of magnitude in momentum scale. Due to the high sensitivity to additional heavy $Z^{\prime}$-bosons (as the contributions of constituents quarks add coherently) APV experiments are competitive to experiments a high energy. In fact, the most stringent lower limit on the mass of an additional $Z^{\prime}$ boson at $1.3 \mathrm{TeV} / \mathrm{c}^{2}$ comes from the Cs APV experiment [11]. APV is furthermore sensitive to additional light neutral gauge bosons, which can decay into light dark matter candidates [13]. Next to being competitive, APV experiments are also complementary to their high-energy counterparts. One example are the constraints on new physics via precision measurements of the coupling constants of the weak neutral-current lepton-quark interactions at low energy, where APV experiments probe an orthogonal band in $\mathrm{C}_{1 u}, \mathrm{C}_{1 d}$ space compared to the parity-violating electron scattering measurements on nuclear targets [14]. Another such example is the ability of experiments to pinpoint the microscopic origin of possible deviations from SM predictions. Correlated effects of SUSY and other SM extensions appear in the $\mathrm{Q}_{\text {weak }}$ in both protons and electrons and can only be separated by comparing to APV experiments [15]. 
The APV signal is strongly enhanced in heavy atoms as is readily observed from the so-called $Z^{3}$ law [16], where $Z$ is the atomic number. A further relativistic correction factor makes that APV is even more strongly enhanced in heavy systems. However, to extract the weak charge and Weinberg angle from experiment, the atomic theory needs to be calculable to high accuracy. The radium ion, $\mathrm{Ra}^{+}$, is a promising candidate for an atomic parity violation (APV) experiment with one single trapped ion [1-4]. The predicted enhancement in $\mathrm{Ra}^{+}$is about 50 times larger than in Cs atoms [4, 17, 18], for which the most accurate measurement has been performed $[6,7,11]$. At the same time, $\mathrm{Ra}^{+}$is still a calculable system: an important ingredient to be able to extract the weak charge from experiment. However, laser spectroscopy on trapped $\mathrm{Ra}^{+}$ions has not been performed yet, and certain spectroscopic information, needed to test the required atomic many-body theory, is lacking [4]. For instance, the lifetimes of the $6^{2} \mathrm{D}_{3 / 2}$ and $6{ }^{2} \mathrm{D}_{5 / 2}$ states, which are important quantities for a single-ion APV experiment, have not been measured yet. These states are also relevant for a potential $\mathrm{Ra}^{+}$optical clock [19-21].

APV manifests itself in $\mathrm{Ra}^{+}$by the tiny admixture of opposite-parity states in the low-lying states. This admixture enables an electric dipole (E1) transition between the the $7^{2} \mathrm{~S}_{1 / 2}$ and $6^{2} \mathrm{D}_{3 / 2}$ state, contrasted by a much stronger parityallowed E2 (or M1) transition. Direct observation of the E1 transition is therefore very challenging. Instead, it is possible to study the inference between the two terms. This measurement principle is quite general in APV experiments [22]. Single trapped ions offer long coherence times and tractable systematics. These properties enable a novel APV measurement technique, based on parity-forbidden AC Stark (or: light) shifts in the ground state Zeeman eigenstates [23] induced by applied laser light fields. This technique involves direct frequency measurements via radiofrequency spectroscopy and subsequent electron shelving. To carry out such a measurements, two standing light waves $E^{\prime}$ and $E^{\prime \prime}$ are applied to off-resonantly drive, respectively, the E1 and E2 transitions between the $7^{2} \mathrm{~S}_{1 / 2}$ and $6{ }^{2} \mathrm{D}_{3 / 2}$ states. The AC Stark shift $\Delta \omega_{\mathrm{APV}}$ due to the interference term can be written as [2]

$$
\Delta \omega_{\mathrm{APV}}=\eta\left\langle 2\left(\mathbf{E}^{\prime} \cdot \nabla\right) \dot{\mathbf{E}}^{\prime \prime}+\mathbf{E}^{\prime} \times\left(\nabla \times \dot{\mathbf{E}}^{\prime \prime}\right)\right\rangle_{t} \cdot \boldsymbol{\sigma},
$$

where $t$ denotes the time average, the dotted vectors stand for time derivatives, $\eta$ is a proportionality constant, and $\sigma$ is the electron spin projection operator. The parityviolating nature of this observable is maybe best explained by comparing the vector containing the electric fields to an effective magnetic field [24] in the sense that the Zeeman eigenstates experience a shift in energy proportional to the $m_{F}$ quantum number. However, this vector transforms like a polar vector under parity (whereas a magnetic field is an axial vector, i.e. P-even). The resulting observable $\Delta \omega_{\mathrm{APV}}$ is P-odd. The largest light shift is caused by the strong E2 transition. However, for suitably chosen light field geometries the E2 contribution does not behave like a polar magnetic field and levels will experience a level shift proportional to $\left|m_{F}\right|$ (cf. Fig. 2). A full study into the systematic effects is warranted as any small $\left(>10^{-3}\right)$ deviation from the ideal geometry will produce APV mimicking effects [2]. This study will be presented in a forthcoming publication. 


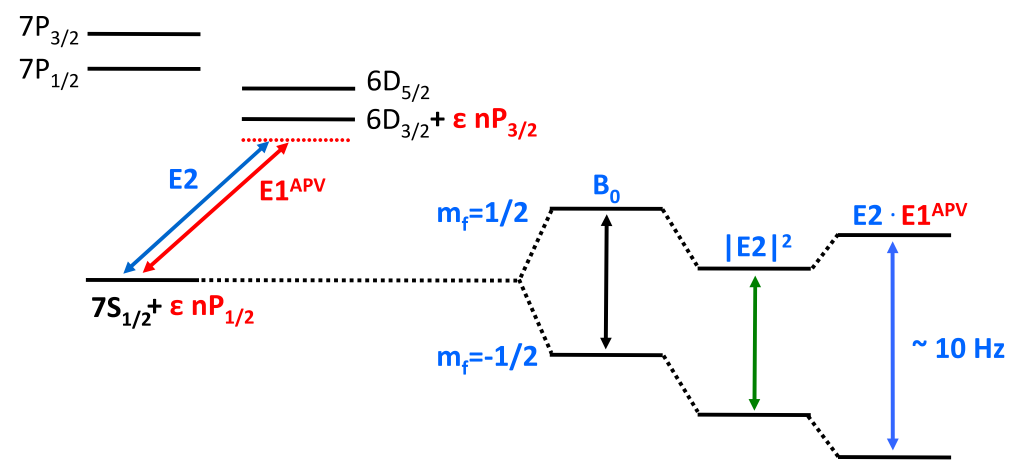

Fig. 2 AC Stark shift in ground state Zeeman eigenstates caused by applied laser light fields. For certain geometries, only the interference term will cause an effective energy difference between $m_{f}=-1 / 2$ and $m_{f}=+1 / 2$, which can be readily observed by RF spectroscopy

\section{Experimental status}

Up to now, accurate experimental information on the optical spectrum of $\mathrm{Ra}^{+}$ (cf. Fig. 3) was only available from measurements at the ISOLDE facility at CERN, where the isotope shift (IS) and hyperfine structure (HFS) of the $7{ }^{2} \mathrm{~S}_{1 / 2}, 7^{2} \mathrm{P}_{1 / 2}$, and $7^{2} \mathrm{P}_{3 / 2}$ states were obtained by collinear spectroscopy over a large range of isotopes $[25,26]$. The only absolute measurement of the relevant wavelengths dates back to arc emission spectroscopy performed on ${ }^{226} \mathrm{Ra}^{+}$in 1933 [27]. We present here the results of on-line excited-state laser spectroscopy experiments of trapped, shortlived ${ }^{212,213,214} \mathrm{Ra}^{+}$ions, obtained at the TRI $\mu \mathrm{P}$ facility [28] of the KVI in Groningen. IS and HFS measurements were performed to constrain the atomic theory: HFS is a sensitive probe of the atomic wave functions in the nucleus [29], the accuracy of which is important for APV, while experiments on different isotopes serve to cancel remaining uncertainties in the atomic theory [4].

\subsection{Experimental setup}

\subsubsection{Production}

Radium isotopes were produced in inverse kinematics by bombarding an 8.5 MeV/nucleon ${ }^{206} \mathrm{~Pb}$ beam of typically $3 \times 10^{10}$ particles/s from the AGOR cyclotron on a $4 \mathrm{mg} / \mathrm{cm}^{2}$ diamond-like carbon foil, and emerged from the fusionevaporation reactions ${ }^{206} \mathrm{~Pb}+{ }^{12} \mathrm{C} \rightarrow{ }^{218-x} \mathrm{Ra}$, in which $x$ neutrons were liberated. The isotopes ${ }^{212} \mathrm{Ra},{ }^{213} \mathrm{Ra}$, and ${ }^{214} \mathrm{Ra}$ were separated from the primary beam and fission products in the magnetic separator [30]. They were stopped and re-ionized to $\mathrm{Ra}^{+}$in a Thermal Ionizer (TI) [28] with a transmission efficiency of $8 \%$. Rates of $800{ }^{212} \mathrm{Ra}^{+} / \mathrm{s}, 2,600{ }^{213} \mathrm{Ra}^{+} / \mathrm{s}$, and $1,000{ }^{214} \mathrm{Ra}^{+} / \mathrm{s}$ were extracted as an ion beam with an energy of $2.8 \mathrm{keV}$. The $\mathrm{Ra}^{+}$isotopes were passed through a Wien Filter (which eliminated contaminants from the TI), and electrostatically decelerated upon injection in a ( $\mathrm{N}_{2}$ or $\mathrm{Ne}$ ) gas-filled Radio Frequency Quadrupole (RFQ) cooler [31], operated at a frequency of $500 \mathrm{kHz}$ with a peak-to-peak RF voltage of $V_{\mathrm{RF}}=380 \mathrm{~V}$ applied between neighboring rods; the opposite half-moon-shaped electrodes, $10 \mathrm{~mm}$ 
Fig. $3{ }^{213} \mathrm{Ra}^{+}$level scheme with wavelengths from [27]. Branching ratios and lifetimes are calculated in [20] (color online)

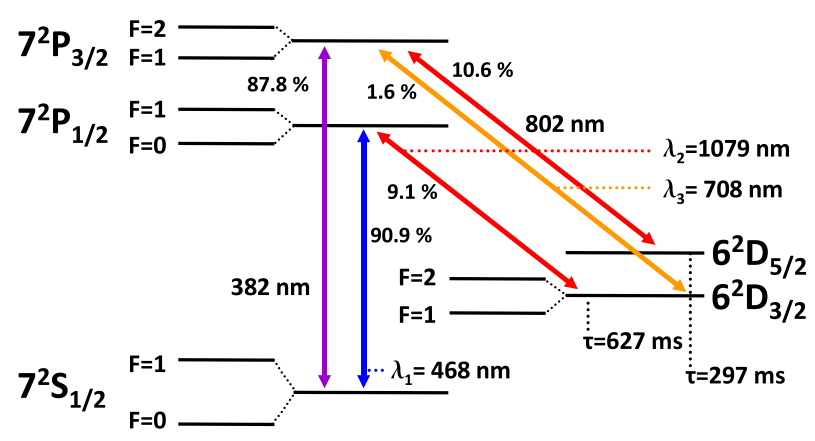

Fig. 4 Schematic overview of the experimental setup behind the RFQ cooler (color online)

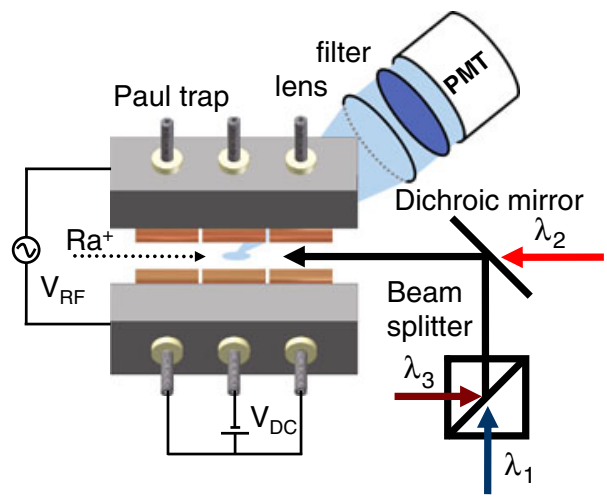

in length, had a tip distance of $5 \mathrm{~mm}$. For on-line optical spectroscopy, the ions were trapped at the end of the RFQ by suitable axial potentials, i.e. a Paul trap (cf. Fig. 4). The effective potential depth was $13 \mathrm{~V}$ while the axial potential depth was $10 \mathrm{~V}$. Typically $10^{3}{ }^{212} \mathrm{Ra}^{+}, 10^{4}{ }^{213} \mathrm{Ra}^{+}$, and $10^{2}{ }^{214} \mathrm{Ra}^{+}$ions could be stored. The storage time was of order $100 \mathrm{~s}$. at a residual gas pressure of $10^{-8}$ mbar (the lifetimes for radioactive decay are $13 \mathrm{~s}, 164 \mathrm{~s}$, and $2.5 \mathrm{~s}$ for ${ }^{212} \mathrm{Ra},{ }^{213} \mathrm{Ra}$, and ${ }^{214} \mathrm{Ra}$, respectively). A $\mathrm{N}_{2}$ or Ne buffer gas was used to aid effective catching and trapping of the radioactive particles from the beam in the RFQ. This gas dissipated the large $(\mathrm{eV})$ energies of the ion beam, compressed the trapped cloud, and also enhanced the storage time. The buffer gas influenced the level lifetimes of the ions because of optical quenching and (hyper)fine-structure mixing of the metastable states. It was expected [32] that $\mathrm{Ne}$ had the smallest influence on the level lifetimes.

\subsubsection{Laser setup}

Home-built Extended Cavity Diode Lasers (ECDLs) were used to drive the optical transitions (cf. Fig. 3). Light to drive the $7^{2} \mathrm{~S}_{1 / 2}-7^{2} \mathrm{P}_{1 / 2}$ transition at wavelength $\lambda_{1}=468 \mathrm{~nm}$ came from NDHA210APAE1 laser diodes from Nichia; the $6{ }^{2} \mathrm{D}_{3 / 2}-$ $7^{2} \mathrm{P}_{1 / 2}$ transition at wavelength $\lambda_{2}=1,079 \mathrm{~nm}$ was driven with light from a LD1080-0075-1 diode from Toptica; the $6{ }^{2} \mathrm{D}_{3 / 2}-7^{2} \mathrm{P}_{3 / 2}$ line at wavelength $\lambda_{3}=708 \mathrm{~nm}$ was excited with light from a HL7001MG diode from Opnext. The laser light was delivered to the ion trap with single-mode optical fibers. The beams were overlapped with polarizing beam splitters and a dichroic mirror and sent axially through the 
Fig. 5 HFS of the $6^{2} \mathrm{D}_{3 / 2}-7^{2} \mathrm{P}_{1 / 2}$ transition in ${ }^{213} \mathrm{Ra}^{+}$. The solid line represents a fit of three Voigt profiles to the data. The Gaussian widths of the resonances are 181(20) $\mathrm{MHz}$ (FWHM). The Lorentzian widths (FWHM) are 245(20), 368(70), and 147(9) MHz (left to right). The different Lorentzian widths are due to saturation effects. The reduced $\chi^{2}=1.1$ at 62 degrees of freedom (dof) (color online)

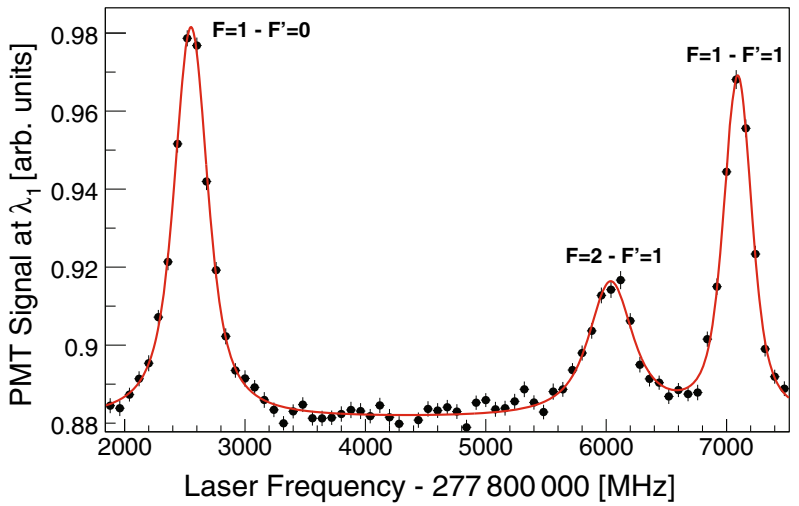

trap to minimize scattered light. They were focussed to $1 \mathrm{~mm}$ diameter at the trap location. Typical laser beam powers $P$ at the trap center were $P\left(\lambda_{1}\right)=300 \mu \mathrm{W}$, $P\left(\lambda_{2}\right)=600 \mu \mathrm{W}$, and $P\left(\lambda_{3}\right)=150 \mu \mathrm{W}$. The wavelengths were monitored with two High-Finesse Angstrom WS6 VIS and IR wavelength meters. Absolute frequency calibration for light at $\lambda_{1}$ was provided by an absorption line in $\mathrm{Te}_{2}$ at wavelength $468.3185 \mathrm{~nm}$ (no. 178 in [33]) through linear absorption in a $\mathrm{Te}_{2}$ glass cell at $450 \mathrm{~K}$. Light at $\lambda_{3}$ was calibrated by linear absorption at the $\mathrm{P}(146)(2-8)$ resonance in $\mathrm{I}_{2}$ in a cell at $500 \mathrm{~K}$. Since for wavelength $\lambda_{2}$ no similar reference was available, it was determined with the IR wavelength meter. The IR wavelength meter was continuously cross-referenced with a cavity of finesse 100 and free spectral range (FSR) $5 \mathrm{GHz}$. The transitions in $\mathrm{Ra}^{+}$were detected through fluorescence light from the $7^{2} \mathrm{~S}_{1 / 2}-7{ }^{2} \mathrm{P}_{1 / 2}$ transition at wavelength $\lambda_{1}$. Because of the $10 \%$ branching into the metastable $6{ }^{2} \mathrm{D}_{3 / 2}$ state, this fluorescence was only observed when both the $7^{2} \mathrm{~S}_{1 / 2}-$ $7^{2} \mathrm{P}_{1 / 2}$ and $6{ }^{2} \mathrm{D}_{3 / 2}-7{ }^{2} \mathrm{P}_{1 / 2}$ transitions were resonantly excited. The fluorescence light was imaged with a single lens of focal length $f=30 \mathrm{~mm}$ inside the vacuum through a low-pass filter with $80 \%$ transmission for wavelengths shorter than $500 \mathrm{~nm}$ (Thorlabs FES0500) onto the photocathode of a photomultiplier (Hamamatsu R7449). The collection solid angle was $0.4 \mathrm{sr}$.

\subsection{Hyperfine structure}

To study the HFS of the $6{ }^{2} \mathrm{D}_{3 / 2}-7{ }^{2} \mathrm{P}_{1 / 2}$ transition in ${ }^{213} \mathrm{Ra}+$ the wavelengths of the light from two diode lasers at $\lambda_{1}$ were kept close to the resonances $7{ }^{2} \mathrm{~S}_{1 / 2} F=1-$ $7^{2} \mathrm{P}_{1 / 2} F^{\prime}=0$ and $7{ }^{2} \mathrm{~S}_{1 / 2} F=0-7{ }^{2} \mathrm{P}_{1 / 2} F^{\prime}=1$. The frequency of the laser light at $\lambda_{2}$ was scanned over the resonances. For this measurement $\mathrm{N}_{2}$ buffer gas was used. Collisions admixed the two hyperfine levels of the $6^{2} \mathrm{D}_{3 / 2}$ level, ensuring that no significant shelving to the metastable $6^{2} \mathrm{D}_{3 / 2} F=1(F=2)$ state occurred when the $6^{2} \mathrm{D}_{3 / 2} F=2(F=1)$ state was depopulated by the resonant laser light at $\lambda_{2}$. The frequency was calibrated with the IR wavelength meter. The measured line shapes are shown in Fig. 5. The different Lorentzian line-widths are due to saturation effects related to various relaxation rates [34], here introduced by the buffer gas. The measured HFS splitting 4542(7) $\mathrm{MHz}$ for the $7^{2} \mathrm{P}_{1 / 2}$ state is within two standard deviations of the value 4525(5) $\mathrm{MHz}$ obtained at ISOLDE [25]. For the $6^{2} \mathrm{D}_{3 / 2}$ state the HFS splitting was measured as $1,055(10) \mathrm{MHz}$; the extracted 
Table 1 HFS constants $A(\mathrm{MHz})$ of the $7^{2} \mathrm{P}_{1 / 2}$ and $6{ }^{2} \mathrm{D}_{3 / 2}$ states in ${ }^{213} \mathrm{Ra}^{+}$

\begin{tabular}{lll}
\hline & $7^{2} \mathrm{P}_{1 / 2}$ & $6^{2} \mathrm{D}_{3 / 2}$ \\
\hline This work & $4,542(7)$ & $528(5)$ \\
ISOLDE [25] & $4,525(5)$ & - \\
Theory [4] & 4,555 & 543 \\
Theory [18] & 4,565 & 541 \\
\hline
\end{tabular}

The most recent theoretical values were converted to ${ }^{213} \mathrm{Ra}^{+}$using the magnetic moment measured at ISOLDE [26]. The theoretical uncertainty is at the \%-level (Timmermans, private communication)

$7^{2} \mathrm{P}_{1 / 2}$ and $6{ }^{2} \mathrm{D}_{3 / 2}$ HFS constants $A$ are given in Table 1 . The theoretical predictions $[4,18]$ are in good agreement with the experimental values.

\subsection{Isotope shift of the $6^{2} \mathrm{D}_{3 / 2}-7^{2} \mathrm{P}_{1 / 2}$ transition}

The IS for the $6{ }^{2} \mathrm{D}_{3 / 2}-7{ }^{2} \mathrm{P}_{1 / 2}$ transition of $\mathrm{Ra}^{+}$was obtained with light from two lasers kept close to wavelength $\lambda_{1}$. One of these laser beams excited the $7^{2} \mathrm{~S}_{1 / 2}-7{ }^{2} \mathrm{P}_{1 / 2}$ transition in ${ }^{212} \mathrm{Ra}^{+}$, while the other one accessed either the $7^{2} \mathrm{~S}_{1 / 2}-$ $7^{2} \mathrm{P}_{1 / 2}$ transition in ${ }^{214} \mathrm{Ra}^{+}$or the $7^{2} \mathrm{~S}_{1 / 2} F=1-7{ }^{2} \mathrm{P}_{1 / 2} F^{\prime}=0$ transition in ${ }^{213} \mathrm{Ra}^{+}$. The frequency of the laser light at $\lambda_{2}$ was scanned over the $6^{2} \mathrm{D}_{3 / 2}-7^{2} \mathrm{P}_{1 / 2}$ resonances of the isotopes under investigation cf. Fig. 6 . The IR wavelength meter was used for frequency calibration. In order to minimize the influence of the buffer gas on the resonance line shape, only $\mathrm{Ne}$ was used. The measurements were performed at gas pressures $3 \times 10^{-4}, 3 \times 10^{-3}$, and $2 \times 10^{-2}$ mbar to study the influence of the buffer gas on the resonance line shapes. No significant effects on the measured IS were found. The resulting IS are summarized in Table 2.

\subsection{Isotope shift of the $6^{2} \mathrm{D}_{3 / 2}-7^{2} \mathrm{P}_{3 / 2}$ transition}

To determine the IS of the $6^{2} \mathrm{D}_{3 / 2}-7^{2} \mathrm{P}_{3 / 2}$ transition the lasers operating at $\lambda_{1}$ and $\lambda_{2}$ were kept close to resonance of a particular $\mathrm{Ra}^{+}$isotope. This created a fluorescence cycle. The frequency of the laser light at $\lambda_{3}$ was scanned over the resonances. Near resonance the ions were pumped to the $7^{2} \mathrm{P}_{3 / 2}$ state, from which some $10 \%$ decayed to the $6{ }^{2} \mathrm{D}_{5 / 2}$ state (cf. Fig. 3 ). In this metastable state the ions were shelved and did not participate in the fluorescence cycle. This caused a dip in the fluorescence signal, the position of which was calibrated against the $\mathrm{P}(146)(2-8)$ single-pass absorption resonance in molecular $\mathrm{I}_{2}$ at $v_{\text {Iodine }}=423433720 \mathrm{MHz}$. The scan linearity was verified with a cavity of finesse 1200 and FSR $10 \mathrm{GHz}$. We found for the $6^{2} \mathrm{D}_{3 / 2}-7{ }^{2} \mathrm{P}_{3 / 2}$ transition $v_{212}=v_{\text {Iodine }}+568(42) \mathrm{MHz}$ for ${ }^{212} \mathrm{Ra}^{+}$ and $v_{214}=v_{\text {Iodine }}+1,269(23) \mathrm{MHz}$ for ${ }^{214} \mathrm{Ra}^{+}$. For ${ }^{213} \mathrm{Ra}^{+}$the fluorescence cycle was established by pumping on the $7^{2} \mathrm{~S}_{1 / 2} F=1-7^{2} \mathrm{P}_{1 / 2} F^{\prime}=0$ transition and repumping on the $6{ }^{2} \mathrm{D}_{3 / 2} F=1-7{ }^{2} \mathrm{P}_{1 / 2} F^{\prime}=0$ transition. This left the $6{ }^{2} \mathrm{D}_{3 / 2} F=2$ state largely depopulated. The frequency of the laser light at $\lambda_{3}$ was scanned over the resonances ( $c f$. Fig. 7). The $6{ }^{2} \mathrm{D}_{3 / 2} F=1-7^{2} \mathrm{P}_{3 / 2} F^{\prime}=1$ resonance is deformed by the close-lying $6{ }^{2} \mathrm{D}_{3 / 2} F=2-7^{2} \mathrm{P}_{3 / 2} F^{\prime}=2$ transition as verified with a rate 
Fig. $66^{2} \mathrm{D}_{3 / 2}-7^{2} \mathrm{P}_{1 / 2}$ resonances in ${ }^{212} \mathrm{Ra}^{+}$and ${ }^{214} \mathrm{Ra}^{+}$. The solid line represents a fit of two Voigt profiles to the data. One parameter is used to fit the Gaussian widths of the two resonances, which yields a FWHM of 436(40) MHz. The Lorentzian widths (FWHM) are 201(50) $\mathrm{MHz}$ and 145(60) $\mathrm{MHz}$ (left to right). The reduced $\chi^{2}=0.92$ at 22 dof (color online)

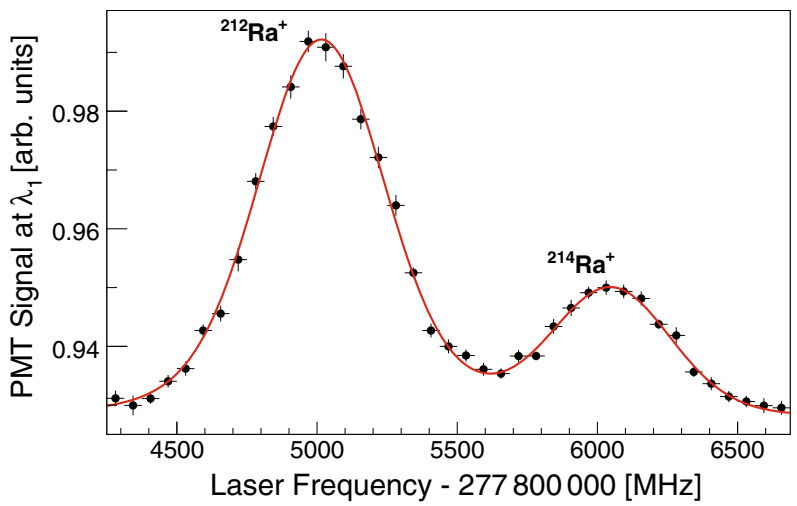

Table 2 IS (MHz) of the $6{ }^{2} \mathrm{D}_{3 / 2}-7{ }^{2} \mathrm{P}_{1 / 2}$ and $6{ }^{2} \mathrm{D}_{3 / 2}-7{ }^{2} \mathrm{P}_{3 / 2}$ transitions in $\mathrm{Ra}+$ isotope pairs

\begin{tabular}{lcll}
\hline & ${ }^{214} \mathrm{Ra}-{ }^{212} \mathrm{Ra}$ & ${ }^{213} \mathrm{Ra}-{ }^{212} \mathrm{Ra}$ & ${ }^{214} \mathrm{Ra}-{ }^{213} \mathrm{Ra}$ \\
\hline $6^{2} \mathrm{D}_{3 / 2}-7^{2} \mathrm{P}_{1 / 2}$ & $1,032(5)$ & $318(11)$ & $714(12)$ \\
$6^{2} \mathrm{D}_{3 / 2}-7^{2} \mathrm{P}_{3 / 2}$ & $701(50)$ & $248(50)$ & $453(34)$ \\
\hline
\end{tabular}

The measured values for the $6{ }^{2} \mathrm{D}_{3 / 2}$ state and a value extracted from $[25,26]$ for the $7^{2} \mathrm{P}_{3 / 2} \mathrm{HFS}$ were used to obtain the IS with respect to the center-of-mass of the resonances in ${ }^{213} \mathrm{Ra}^{+}$

equation model. We use the $6{ }^{2} \mathrm{D}_{3 / 2} F=2-7{ }^{2} \mathrm{P}_{3 / 2} F^{\prime}=1$ resonance to determine the IS. The measurements were carried out at gas pressures $3 \times 10^{-4}, 2 \times 10^{-3}$, and $2 \times 10^{-2}$ mbar. The power of the laser beam at $\lambda_{3}$ was varied between 50 and $150 \mu \mathrm{W}$; no significant changes were found. We found $v_{213}=v_{\text {Iodine }}-64(13) \mathrm{MHz}$. The measured isotope shifts are summarized in Table 2. The absolute frequency of the $6{ }^{2} \mathrm{D}_{3 / 2}-7{ }^{2} \mathrm{P}_{3 / 2}$ transition in ${ }^{212} \mathrm{Ra}^{+}$is $423434288(42) \mathrm{MHz}$. It had earlier been measured to be $423437660(570) \mathrm{MHz}$ [27] for ${ }^{226} \mathrm{Ra}^{+}$, which yields an IS of 3.4(6) GHz.

\subsection{Lifetime of the $6^{2} \mathrm{D}_{5 / 2}$ state}

The demonstrated shelving to the $6^{2} \mathrm{D}_{5 / 2}$ state by accessing the $6^{2} \mathrm{D}_{3 / 2}-$ $7^{2} \mathrm{P}_{3 / 2}$ transition also enables a measurement of the lifetime of this metastable state. The lasers at $\lambda_{1}$ and $\lambda_{2}$ were kept close to resonance in ${ }^{212} \mathrm{Ra}^{+}$, while the laser light at $\lambda_{3}$ was pulsed with $170 \mathrm{~ms}$ on-periods and $670 \mathrm{~ms}$ off-periods by a mechanical chopper wheel. The laser light at $\lambda_{3}$ was kept on resonance to populate $6{ }^{2} \mathrm{D}_{5 / 2}$ via the $7^{2} \mathrm{P}_{3 / 2}$ state. When the laser light at $\lambda_{3}$ was switched off, the $6{ }^{2} \mathrm{D}_{5 / 2}$ state depopulated and the ions re-entered the fluorescence cycle with a time constant equal to the lifetime of the $6^{2} \mathrm{D}_{5 / 2}$ state (cf. Fig. 8). However, the neon buffer gas caused a reduction of the lifetime of the metastable state by quenching it to the ground state and by means of fine structure mixing between the the $6{ }^{2} \mathrm{D}_{5 / 2}$ and $6{ }^{2} \mathrm{D}_{3 / 2}$ states. The latter effect causes the $6^{2} \mathrm{D}_{5 / 2}$ state to be pumped out by the the laser light at $\lambda_{2}$ via the $6^{2} \mathrm{D}_{3 / 2}$ state. To estimate the effects of the buffer gas, measurements were conducted at different gas pressures ranging from $10^{-2}$ to $10^{-5} \mathrm{mbar}$. The buffer gas 


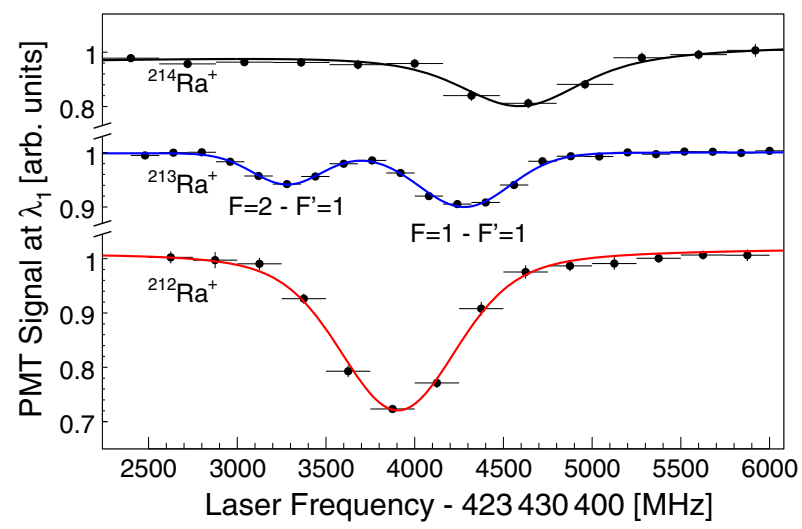

Fig. 7 The $6{ }^{2} \mathrm{D}_{3 / 2}-7^{2} \mathrm{P}_{3 / 2}$ transitions. The solid lines represent fits of Voigt profiles to the data with reduced $\chi^{2}$ 's of $0.81,0.81$, and 0.88 for ${ }^{212} \mathrm{Ra}^{+},{ }^{213} \mathrm{Ra}^{+}$, and ${ }^{214} \mathrm{Ra}^{+}$, respectively, at 18,26 , and 17 dof. The corresponding Gaussian and Lorentzian widths (FWHM) are 655(12) MHz and 243(10) $\mathrm{MHz}$, respectively, for ${ }^{212} \mathrm{Ra}^{+}, 363(30)$ and $144(23) \mathrm{MHz}$ for the $F=2-F^{\prime}=1$ transition in ${ }^{213} \mathrm{Ra}^{+}$, and 451(270) $\mathrm{MHz}$ and 581(300) $\mathrm{MHz}$ for ${ }^{214} \mathrm{Ra}^{+}$. The different Gaussian widths are due to different neon buffer gas pressures. The different Lorentzian widths are caused by saturation effects which vary with gas pressure and laser power (color online)

Fig. 8 Lifetime measurements of the ${ }^{212} \mathrm{Ra}^{+} 6^{2} \mathrm{D}_{5 / 2}$ state at a neon buffer gas pressure of $4 \times 10^{-5}$ mbar. The solid line represents a fit of an exponential function to the data. The fit yields a lifetime of 232(4) ms with a $\chi^{2}=0.83$ at 87 dof

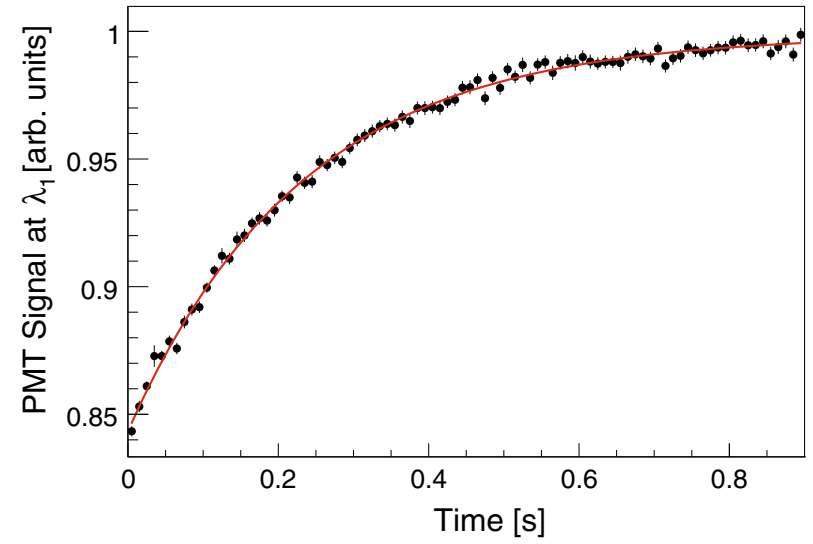

was shown to have a strong influence on the optical lifetime (cf. Fig. 9). Assuming a linear dependence of the mixing rates on the gas pressure [32], the buffer gas pressure $\mathrm{P}_{\mathrm{Ne}}$ dependence is

$$
\tau \tau_{m}=\left[\frac{1}{\tau_{n}}+\sum_{i} \alpha_{i} P_{i}\right]^{-1},
$$

where $\tau_{m}$ is the measured lifetime, $\tau_{n}$ is the natural radiative lifetime, $\alpha_{i}$ is the mixing or quenching rate per millibar of gas, for gas of type $i$ of which $P_{i}$ is the partial pressure. Residual partial pressures of other gases were measured below a few $10^{-7}$ mbar, and are neglected. The fit of (2) to the data yields $\tau_{n}=231$ (4) $\mathrm{ms}$, with a reduced $\chi^{2}=6.1$ at 2 dof. The mixing or quenching rate constant is found 
Fig. 9 Lifetime of the $\mathrm{Ra}^{+}$ $6^{2} \mathrm{D}_{5 / 2}$ state as a function of neon buffer gas pressure. The solid line represents a fit of (2). The fit yields a $\chi^{2}=6.1$ at 2 dof

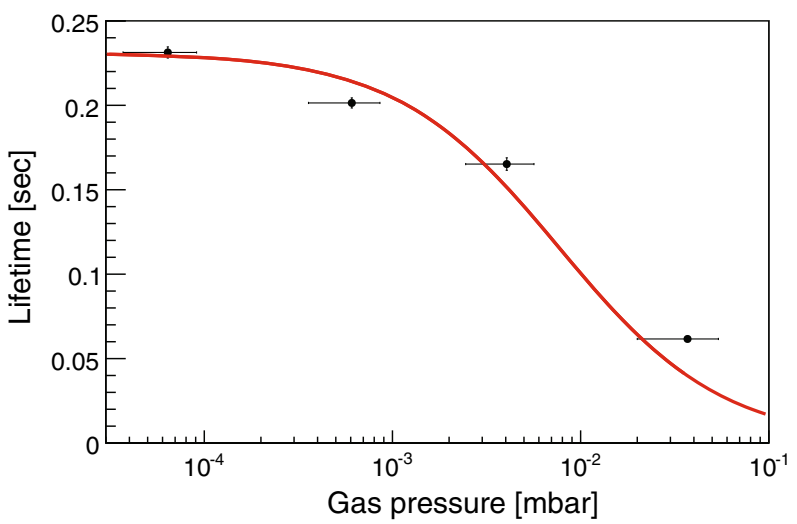

to be $\alpha_{\mathrm{Ne}}=562(105) \mathrm{mbar}^{-1} \mathrm{~s}^{-1}$ or $2.2(4) \times 10^{-14} \mathrm{~cm}^{3} \mathrm{~s}^{-1}$. This rate is an order of magnitude lower than rates measured for other ions [32]. This can be explained by the strong dependence of the mixing rates on the fine structure splitting (i.e. $6^{2} \mathrm{D}_{5 / 2}-$ $6^{2} \mathrm{D}_{3 / 2}$ ) [35], which is larger in radium than in the aforementioned other ions. The quenching rate is generally some two orders of magnitude smaller than the mixing rate [32]. The 5\% confidence level corresponding to the fit indicates that the linear dependence breaks down over this large pressure range. No further detailed theory for this system is presently available to extrapolate the lifetime to zero pressure. A lower bound on the radiative lifetime of the $6^{2} \mathrm{D}_{5 / 2}$ state was found to be $232(4) \mathrm{ms}$; it corresponds to the lifetime measured at the lowest pressure of some $4 \times 10^{-5} \mathrm{mbar}$ (cf. Fig. 8). Corrections for the radioactive lifetime of ${ }^{212} \mathrm{Ra}$ and for the replacement time can be neglected. Theoretical predictions are 297(4) ms [20] and 303(4) ms [18]. Our experimental result is an important confirmation that the $6^{2} \mathrm{D}_{5 / 2}$ state is indeed long-lived. This is a necessary property in view of the long coherence times needed in APV experiments with a single trapped ion [1].

\section{Conclusions}

Atomic parity violation (APV) experiments are sensitive probes of the electroweak interaction at low energy. These experiments are competitive with and complementary to high-energy collider experiments. To provide much needed experimental input, for the first time on-line excited-state laser spectroscopy was performed on short-lived trapped ions. HFS measurements are suited to test wave functions at the origin, whereas the measurement of radiative lifetimes test them at larger distances. IS measurements probe atomic theory and yield information about the size and shape of the atomic nucleus. These measurements test the atomic theory, the accuracy of which is indispensable for upcoming single-ion APV experiments aiming at an improved low-energy determination of the electroweak mixing angle [4]. For the refinement of this test, Ra offers a chain of isotopes, where no measurements have been made and where theory is challenged to provide unbiased predictions. 
Acknowledgements We acknowledge the support received from the AGOR cyclotron group and the KVI technical personnel. O. Böll, O. Dermois, and L. Huisman were essential in the design and the setup of the experiment. We thank R. Hoekstra for useful discussions. This research was supported by the Stichting voor Fundamenteel Onderzoek der Materie (FOM) under Program 114 (TRI $\mu \mathrm{P})$ and FOM projectruimte 06PR2499. O.O.V. acknowledges funding from the NWO Toptalent program and ITS LEIF.

Open Access This article is distributed under the terms of the Creative Commons Attribution Noncommercial License which permits any noncommercial use, distribution, and reproduction in any medium, provided the original author(s) and source are credited.

\section{References}

1. Fortson, N.: Phys. Rev. Lett. 70, 2383 (1993)

2. Koerber, T.W., et al.: J. Phys. B 36, 637 (2003)

3. Sherman, J.A., Koerber, T.W., Markhotok, A., Nagourney, W., Fortson, E.N.: Phys. Rev. Lett. 94, 243001 (2005)

4. Wansbeek, L.W., et al.: Phys. Rev. A 78, 050501(R) (2008)

5. Bouchiat, M.A., et al.: Phys. Lett. B 117(5), 358 (1982)

6. Wood, C.S., et al.: Science 275, 1759 (1997)

7. Bennett, S.C., Wieman, C.E.: Phys. Rev. Lett. 82, 2484 (1999)

8. Gwinner, G., et al.: Hyperfine Interact. 172, 45 (2006)

9. Stancari, G., et al.: Eur. Phys. J. Spec. Top. 150, 389 (2007)

10. Tsigutkin, K., et al.: Phys. Rev. Lett. 103(7), 071601 (2009)

11. Porsev, S.G., Beloy, K., Derevianko, A.: Phys. Rev. Lett. 102(18), 181601 (2009)

12. Hasert, F.J., et al.: Phys. Lett. B46, 138 (1973). doi:10.1016/0370-2693(73)90499-1

13. Bouchiat, C., Fayet, P.: Phys. Lett. B 608(1-2), 87 (2005). doi:10.1016/j.physletb.2004.12.065. URL: http:/www.sciencedirect.com/science/article/B6TVN-4F6D65D-1/2/c27e1d9445bdde6b7 af17e2d9a387fea

14. Young, R.D., Carlini, R.D., Thomas, A.W., Roche, J.: Phys. Rev. Lett. 99(12), 122003 (2007). doi:10.1103/PhysRevLett.99.122003

15. Ramsey-Musolf, M., Su, S.: Phys. Rep. 456(1-2), 1 (2008). doi:10.1016/j.physrep.2007.10.001. URL: http://www.sciencedirect.com/science/article/B6TVP-4PW5XH2-1/2/d3f2faf0b6a9d80b5b4 5a1c510f73d55

16. Bouchiat, M.A., Bouchiat, C.C.: Phys. Lett. B 48, 111 (1974)

17. Dzuba, V.A., Flambaum, V.V., \& Ginges, J.S.M.: Phys. Rev. A 63, 062101 (2001)

18. Pal, R., Jiang, D., Safronova, M.S., Safronova, U.I.: Phys. Rev. A 79(6), 062505 (2009). doi:10.1103/PhysRevA.79.062505

19. Dzuba, V.A., Flambaum, V.V.: Phys. Rev. A 61, 034502 (2000)

20. Sahoo, B.K., et al.: Phys. Rev. A 76, 040504(R) (2007)

21. Sahoo, B.K., Timmermans, R.G.E., Das, B.P., Mukherjee, D.: Phys. Rev. A 80, 062506 (2009)

22. Guéna, J., Lintz, M., Bouchiat, M.A.: Mod. Phys. Lett. A 20, 375 (2005)

23. Fortson, N.: Phys. Rev. Lett. 70(16), 2383 (1993). doi:10.1103/PhysRevLett.70.2383

24. Happer, W., Mathur, B.S.: Phys. Rev. 163(1), 12 (1967). doi:10.1103/PhysRev.163.12

25. Wendt, K., et al.: Z. Phys. D 4(19-20), 227 (1987). doi:10.1007/BF01436633

26. Arnold, E., et al.: Phys. Rev. Lett. 59(7), 771 (1987). doi:10.1103/PhysRevLett.59.771

27. Rasmussen, E.: Z. Phys. 86, 24 (1933)

28. Shidling, P.D., et al.: Nucl. Instrum. Methods A 606(3), 305 (2009). doi:10.1016/j.nima.2009. 05.007

29. Dinh, T.H., Dzuba, V.A., Flambaum, V.V.: Phys. Rev. A 80(4), 044502 (2009). doi:10.1103/ PhysRevA.80.044502

30. Berg, G.P.A., et al.: Nucl. Instrum. Methods A 560(2), 169 (2006)

31. Traykov, E., et al.: Nucl. Instrum. Methods B 266(19-20), 4532 (2008). doi:10.1016/j.nimb. 2008.05.077

32. Knoop, M., Vedel, M., Vedel, F.: Phys. Rev. A 58(1), 264 (1998). doi:10.1103/PhysRevA.58.264

33. Cariou, J., Luc, P.: Atlas du Spectre d'Absorption de la Molécule de Tellure. CNRS, Paris (1980)

34. Budker, D., Kimball, D.F., DeMille, D.P.: Atomic Physics. Oxford University Press, London (2008)

35. Knoop, M., Vedel, M., Vedel, F.: Phys. Rev. A 52(5), 3763 (1995). doi:10.1103/PhysRevA.52.3763 